\title{
LEAF NUTRITIONAL LEVELS IN PEACH AND NECTARINE GROWN IN SUBTROPICAL CLIMATE ${ }^{1}$
}

\author{
SARITA LEONEL ${ }^{2}$, MANOEL EUZÉBIO DE SOUZA², MARCO ANTONIO TECCHIO ${ }^{3}$, \\ DANIELA MOTA SEGANTINI ${ }^{2}$
}

ABSTRACT - The study evaluated the leaf nutritional levels of peach and nectarine trees under subtropical climate in order to improve the fertilization practices. The experiment was carried out in São Paulo state University, Botucatu, São Paulo State, Brazil. The experimental design consisted of subdivided plots, in which plots corresponded to cultivars and subplots to the leaf sample periods. The evaluated peach cultivars were: Marli, Turmalina, Precocinho, Jubileu, Cascata 968, Cascata 848, CP 951C, CP 9553CYN, and Tropic Beauty, and that of nectarine was 'Sun Blaze'. The sample periods were: after harvest, plants in vegetative period; dormancy; beginning of flowering and fruiting (standard sample). Results indicated significant variations in the levels of $\mathrm{N}, \mathrm{P}, \mathrm{K}, \mathrm{Ca}, \mathrm{Mg}, \mathrm{S}, \mathrm{B}, \mathrm{Cu}, \mathrm{Fe}, \mathrm{Mn}$ and $\mathrm{Zn}$ for the sampling period and in $\mathrm{N}, \mathrm{Ca}$, $\mathrm{Mg}, \mathrm{S}, \mathrm{B}, \mathrm{Fe}$ and Mn levels for the cultivars.

Index terms: Prunus persica var. vulgaris, Prunus persica var. nucipersica, mineral nutrition, phenology.

\section{TEORES NUTRICIONAIS FOLIARES EM PESSEGUEIRO E NECTARINEIRA CULTIVADOS EM CLIMA SUBTROPICAL}

RESUMO - O trabalho teve como objetivo avaliar os teores foliares de nutrientes das cultivares de pessegueiro e nectarineira, cultivados em clima subtropical, visando a fornecer subsídios às práticas de adubação da cultura nessas condições. O experimento foi realizado na Faculdade de Ciências Agronômicas, UNESP, Câmpus de Botucatu. O delineamento experimental empregado foi em parcelas subdivididas, em que ás parcelas corresponderam às cultivares avaliadas e as subparcelas às épocas de coleta das amostras foliares. As cultivares de pessegueiro avaliadas foram: Marli, Turmalina, Precocinho, Jubileu, Cascata 968, Cascata 848, CP 951-C, CP 9553-CYN e Tropic Beauty e da nectarineira 'Sun Blaze'. As épocas de coleta das amostras foram: 1 - após a colheita dos frutos, no período vegetativo (fevereiro); 2 - na dormência (maio); 3 - início do florescimento (agosto), e $4-13^{\mathrm{a}}$ a $15^{\mathrm{a}}$ semana após a plena floração, antes da colheita - frutificação (época padrão - outubro-novembro). Os resultados obtidos permitiram concluir que houve variações significativas para os teores de $\mathrm{N}, \mathrm{P}, \mathrm{K}, \mathrm{Ca}, \mathrm{Mg}, \mathrm{S}, \mathrm{B}, \mathrm{Cu}, \mathrm{Fe}, \mathrm{Mn}$ e $\mathrm{Zn}$ em relação às épocas de amostragem e para N, Ca, $\mathrm{Mg}, \mathrm{S}, \mathrm{B}, \mathrm{Fe}$ e $\mathrm{Mn}$, para os teores nutricionais nas cultivares.

Termos para indexação: Prunus persica var. vulgaris, Prunus persica var. nucipersica, nutrição mineral, fenologia.

\section{INTRODUCTION}

Peach production in Brazil was 239,149 tons in 2008, and the harvested area was 21,326 ha (ANUÁRIO BRASILEIRO DA FRUTICULTURA, 2010). Located in a warmer climatic region, São Paulo State contributes with around $10 \%$ planted area and 23\% national production, presenting 21.5 $\mathrm{t} / \mathrm{ha} /$ year mean productivity, as well as economic potential for the cultivation of peach and nectarine trees. The state has the early fruit harvest as its great advantage not only over the main Brazilian producing regions, but also over most countries located in the Southern Hemisphere, such as Chile, Argentina, Uruguay and South Africa (PEREIRA et al., 2002).

In subtropical regions, specific procedures for orchard management are necessary, especially nutritional control, in which balanced and satisfactory mineral nutrition factors are related to good harvests at the stages of plant production and formation. Fructification and production for most temperate fruit plants, including peach trees, are

\footnotetext{
${ }^{1}$ Trabalho Sinfruit 074 - Simpósio Internacional de Fruticultura - Avanços na Fruticultura (17 a 21 Outubro)

${ }^{2}$ UNESP. Faculdade de Ciências Agronômicas. Departamento de Produção Vegetal/Horticultura. Rua José Barbosa de Barros, 1780. CEP 18610-307. Botucatu-SP. E-mail: sarinel@fca.unesp.br.

${ }^{3}$ Centro Avançado de Pesquisa Tecnológica do Agronegócio de Frutas - Instituto Agronômico de Campinas.
} 
initially associated with the suitable development of flower buds. Thus, nutritional and phytosanitary management, as well as suitable environmental conditions to break dormancy, are essential (NAVA et al., 2009). An adequate nutritional status of flowers leads to higher and effective fructification for most fruit species, and nutritional competition is also frequently associated with low fructification levels in several fruit trees. The competition for nutrients during flower bud differentiation originates malformed flowers, negatively affecting fructification (CROSSA-RAYNAUD et al., 1985).

Early defoliation is a serious and very common problem in the main peach producing regions in Brazil, especially in São Paulo State, where the temperatures of the cultivated areas are higher. According to Nava et al. (2009), early defoliation, caused by either environmental and nutritional issues or phytosanitary problems, usually decreases carbohydrate levels in the plants, affecting their fructification. Some reports in the literature consider that a suitable nutritional status of flower buds may overcome the effect of high temperatures during flowering due to the prolongation of the pistil receptivity time (GAO et al., 2002 and WOLUKAU et al., 2004).

Since nutritional management of peach and nectarine trees cultivated in subtropical regions is still little known, especially regarding leaf fertilization and different cultivar requirements, this study aimed to evaluate the leaf nutritional content for peach and nectarine cultivars at different phenological stages over the production cycle in order to provide information for fertilization techniques under those conditions.

\section{MATERIAL AND METHODS}

This experiment was carried out in the School of Agronomical Sciences, São Paulo State University (UNESP), Botucatu Municipality, São Paulo State, Brazil, at $22^{\circ} 51^{\prime} 55^{\prime \prime} \mathrm{S}, 48^{\circ} 27^{\prime} 22^{\prime \prime}$ $\mathrm{W}$ and $810 \mathrm{~m}$ altitude. The climate of this region is mesothermal, $C w a$, i.e. subtropical humid including drought during the winter and rain from November to April; mean annual precipitation is $1,433 \mathrm{~mm}$ and mean annual temperature, $19.3{ }^{\circ} \mathrm{C}$ (Cunha et al., 1999). The soil was classified as Red Nitosol, according to Embrapa's criteria (1996). At 0-20cm depth, the following chemical characteristics were detected for the soil: $\mathrm{pH}\left(\mathrm{CaCl}_{2}\right)=5.4 ; \mathrm{OM}=35$ $\mathrm{g} \mathrm{dm}^{-3} ; \mathrm{P}($ resin $)=16 \mathrm{mg} \mathrm{dm}^{-3} ; \mathrm{H}+\mathrm{Al}=27 \mathrm{mmol}_{\mathrm{c}}$ $\mathrm{dm}^{-3} ; \mathrm{K}=3.7 \mathrm{mmol} \mathrm{dm}_{\mathrm{c}}^{-3} ; \mathrm{Ca}=28 \mathrm{mmol}_{\mathrm{c}} \mathrm{dm}^{-3}$; $\mathrm{Mg}=13 \mathrm{mmol}_{\mathrm{c}} \mathrm{dm}^{-3}$; $\mathrm{SB}=45 \mathrm{mmol}_{\mathrm{c}} \mathrm{dm}^{-3}$; CEC
$=72 \mathrm{mmol}_{\mathrm{c}} \mathrm{dm}^{-3} ; \mathrm{V}=63 \%$, which were analyzed according to RAIJ \& QUAGGIO (1983).

The plants from the experimental area aged 7 years and were spaced $6.0 \mathrm{~m}$ between plants $\mathrm{x}$ $4.0 \mathrm{~m}$ between rows, in a dryland area. The rootstock 'Okinawa' was used for all canopy cultivars. To assure the good development of plants, cultural and phytosanitary procedures were performed during the whole experimental period by following technical recommendations (PEREIRA et al., 2002).

Samples of recently mature complete leaves (blade with petiole) were harvested from the medium portion of current-year branches in the different plant quadrants (Malavolta et al., 1997). Eight leaves were harvested from each plant, totaling three plants per plot, with four replicates. The total sample was composed of 96 leaves for each evaluated cultivar. The levels of the nutrients $\mathrm{N}, \mathrm{P}, \mathrm{K}, \mathrm{Ca}$, $\mathrm{Mg}, \mathrm{S}, \mathrm{B}, \mathrm{Cu}, \mathrm{Fe}, \mathrm{Mn}$, and $\mathrm{Zn}$ were quantified, according to the methodology proposed by Malavolta et al. (1997), in the Laboratory of Plant Mineral Nutrition. Harvests occurred: 1 - after fruit harvest, at the vegetative stage (February); 2 - during the dormancy period (May); 3 - at flowering beginning (August); and 4 - from the $13^{\text {th }}$ to the $15^{\text {th }}$ week after full flowering, before harvest - fructification (October-November); this period is considered ideal to compare leaf patterns (LEECE \& BARKS, 1974; JOHSON \& URIU, 1989; FREIRE \& MAGNANI, 2003).

The peach cultivars Marli, Precocinho, Turmalina, Jubileu, Cascata 968, Cascata 848, CP 951-C, CP 9553-CYN, and Tropic Beauty, as well as the nectarine cultivar 'Sun Blaze', were evaluated.

The experimental design was subdivided plots, in which plots corresponded to the cultivars evaluated, and the subplots, to the leaf harvests in 2009. The obtained results were subjected to analysis of variance and, as for significance, means were compared by Tukey's test at 1 and 5\% probability.

\section{RESULTS AND DISCUSSION}

There were significant variations in the levels of $\mathrm{N}, \mathrm{P}, \mathrm{K}, \mathrm{Ca}, \mathrm{Mg}, \mathrm{S}, \mathrm{B}, \mathrm{Cu}, \mathrm{Fe}, \mathrm{Mn}$, and $\mathrm{Zn}$ according to harvests (Tables 1 to 6), which confirms that the uptake of mineral elements by peach trees varies with the plant physiological stages over the production cycle. The highest levels of N, S, B, and Zn were detected at the third harvest, corresponding to the onset of flowering. As regards P levels, the highest values were obtained at the third (flowering onset) and fourth (fructification) harvests. $\mathrm{K}$ and $\mathrm{Ca}$ levels were highest in the fructification period 
(fourth harvest), which corroborates the statement that these elements are essential to fruit quality in this period. The highest $\mathrm{Mg}$ and Mn levels were observed in the dormancy period (second harvest). At the first (after collection) and third (flowering onset) harvests, $\mathrm{Cu}$ levels were highest. Fe was highest at the second (dormancy) and third (flowering onset) harvests.

Johnson et al. (2006) emphasize that, although leaf sampling is worldwide recommended during the fructification stage, when the requirement for most nutrients is highest since fruits are the strongest plant drains, other periods can lead to better uptake of different nutrients, as observed in the present study. These authors also reported that leaf sampling during fructification can be considered very late to fulfill the fertilization requirements of the annual growth season. Based on this statement, the same authors evaluated leaf harvest during the dormancy period and observed a good response for $\mathrm{N}, \mathrm{P}, \mathrm{B}$, and $\mathrm{Zn}$. In their opinion, the other nutrients did not present response because deficiency symptoms had not been induced yet.

As regards the leaf content considered suitable for peach and nectarine trees, Leece \& Barks (1974) reported the following international patterns: $\mathrm{N}(3.0-3.5 \%), \mathrm{P}(0.14-0.25 \%), \mathrm{K}(2.0-3.0 \%), \mathrm{Ca}$ $(1.8-2.7 \%), \mathrm{Mg}(0.30-0.80 \%), \mathrm{Na}(<0.02 \%), \mathrm{Cl}$ $(<0.3 \%), \mathrm{Fe}\left(100-250 \mu \mathrm{g} \mathrm{g}^{-1}\right), \mathrm{Cu}\left(5-16 \mu \mathrm{g} \mathrm{g}^{-1}\right), \mathrm{Mn}$ $\left(40-160 \mu \mathrm{g} \mathrm{g}^{-1}\right), \mathrm{Zn}\left(20-50 \mu \mathrm{g} \mathrm{g}^{-1}\right)$, and B (15-19 $\left.\mu \mathrm{g} \mathrm{g}^{-1}\right)$. Johnson \& Uriu (1989), in the United States, reported the following levels as optimal range: $\mathrm{N}$ (2.6-3.0\%), P (0.1-0.3\%), K (>1.2\%), Ca (>1.0\%), $\mathrm{Mg}(>0.25 \%), \mathrm{Fe}(>60 \mathrm{ppm}), \mathrm{Mn}(>20 \mathrm{ppm}), \mathrm{Zn}$ (>20 ppm), B (20-80 ppm), and $\mathrm{Cu}(>4 \mathrm{ppm})$. The Soil Fertility Commission of Rio Grande do Sul State, Brazil (1994), set normal nutritional ranges for peach leaf samples, at the following levels: $\mathrm{N}$ (3.24-4.53\%), P (0.15-0.28\%), K (1.31-2.06\%),
$\mathrm{Ca}(1.64-2.61 \%), \mathrm{Mg}(0.52-0.83 \%), \mathrm{Fe}(100-230$ $\left.\mathrm{mg} \mathrm{kg}{ }^{-1}\right), \mathrm{Mn}\left(31-160 \mathrm{mg} \mathrm{kg}^{-1}\right), \mathrm{Zn}\left(24-37 \mathrm{mg} \mathrm{kg}^{-1}\right)$, $\mathrm{Cu}\left(6-30 \mathrm{mg} \mathrm{kg}^{-1}\right)$, and $\mathrm{B}\left(34-63 \mathrm{mg} \mathrm{kg}^{-1}\right)$.

The present results for the levels of N, P, K, $\mathrm{Cu}, \mathrm{Mn}, \mathrm{Zn}$, and $\mathrm{Fe}$ were within the normal range, although there were differences regarding leaf harvests. $\mathrm{Ca}$ and $\mathrm{Mg}$ levels were below the recommended patterns, whereas B levels were above those values. $\mathrm{S}$ levels could not be compared since there were no reports on this element in the literature.

As regards nutrient uptake by the different evaluated cultivars, there were significant variations in the levels of $\mathrm{N}, \mathrm{Ca}, \mathrm{Mg}, \mathrm{S}, \mathrm{B}, \mathrm{Fe}$, and $\mathrm{Mn}$, with different responses according to the nutrient. In general, leaf macronutrient uptake by the evaluated cultivars was in the following order: $\mathrm{N}>\mathrm{K}>\mathrm{Ca}>\mathrm{P}>\mathrm{Mg}>\mathrm{S}$. For micronutrients, the order was: $\mathrm{Fe}>\mathrm{Mn}>\mathrm{B}>\mathrm{Zn}>\mathrm{Cu}$. According to Fachinello et al. (1996), nutrient export $(\mathrm{kg} / \mathrm{t})$ by fruits, pruned branches and leaves is: $\mathrm{N}(3.5), \mathrm{P}_{2} \mathrm{O}_{5}(1.05)$ and $\mathrm{K}_{2} \mathrm{O}$ (5.5), without considering the growth of peach trees. Malavolta (1980) reported that the levels of macronutrients $(\mathrm{kg} / \mathrm{ha})$ exported over peach fruit harvests (kg/ha) are: N (70); P(6); K (40); S (4); $\mathrm{Ca}$ (1.2); and $\mathrm{Mg}$ (2.4).

The results of the present study agree with those in the specialized literature, which state that nitrogen presents the highest uptake and the highest influence on peach tree productivity (Rombolá et al., 2000) since it directly affects the vegetative branch growth (Mattos et al., 1991), the number of vegetative and flower buds, and the number of fruits per plant. This nutrient can provide a longer period of leaf permanence, consequently increasing the period of reserve accumulation for the subsequent cycle (SERRAT et al., 2004). However, excessive supply can lead to oversprouting, which results in excessive shading, decreasing the fruit exposure to sunlight (MATTOS et al., 1991). 
TABLE 1 - Nitrogen and phosphorus levels $\left(\mathrm{g} \mathrm{kg}^{-1}\right)$ in leaf samples of peach and nectarine cultivars at different harvests. Botucatu, 2009.

\begin{tabular}{|c|c|c|c|c|c|}
\hline \multirow[b]{2}{*}{ CULTIVARS } & \multicolumn{5}{|c|}{ HARVESTS } \\
\hline & 1 & 2 & 3 & 4 & Mean \\
\hline & \multicolumn{5}{|c|}{ Nitrogen levels $\left(\mathrm{g} \mathrm{kg}^{-1}\right)$} \\
\hline Turmalina & $43 \mathrm{Aa}$ & $35 \mathrm{ABb}$ & $47 \mathrm{Ba}$ & $34 \mathrm{ABCb}$ & $40 \mathrm{~A}$ \\
\hline Cascata 968 & $39 \mathrm{ABCa}$ & $30 \mathrm{Bb}$ & $42 \mathrm{BCa}$ & $32 \mathrm{ABCb}$ & $36 \mathrm{AB}$ \\
\hline Cascata 848 & $37 \mathrm{ABCb}$ & $36 \mathrm{ABb}$ & $45 \mathrm{BCa}$ & $34 \mathrm{ABCb}$ & $38 \mathrm{AB}$ \\
\hline Precocinho & $40 \mathrm{ABb}$ & $39 \mathrm{Ab}$ & $48 \mathrm{ABa}$ & $28 \mathrm{Cc}$ & $39 \mathrm{~A}$ \\
\hline CP 9553 & $37 \mathrm{ABCb}$ & $32 \mathrm{ABbc}$ & $44 \mathrm{BCa}$ & $29 \mathrm{BCc}$ & $36 \mathrm{AB}$ \\
\hline СР 951 & $32 \mathrm{Cb}$ & $31 \mathrm{Bb}$ & $39 \mathrm{Ca}$ & $32 \mathrm{ABCb}$ & $34 \mathrm{~B}$ \\
\hline Marli & $39 \mathrm{ABCb}$ & $31 \mathrm{Bc}$ & $47 \mathrm{BCa}$ & $37 \mathrm{Ab}$ & $38 \mathrm{~A}$ \\
\hline Tropic Beauty & $32 \mathrm{Cb}$ & $34 \mathrm{ABb}$ & $44 \mathrm{BCa}$ & $36 \mathrm{ABb}$ & $37 \mathrm{AB}$ \\
\hline Sun Blaze & $34 \mathrm{BCb}$ & $31 \mathrm{Bb}$ & $56 \mathrm{Aa}$ & $33 \mathrm{ABCb}$ & $38 \mathrm{~A}$ \\
\hline Jubileu & $34 \mathrm{BCb}$ & $32 \mathrm{ABb}$ & $43 \mathrm{BCa}$ & $34 \mathrm{ABCb}$ & $36 \mathrm{AB}$ \\
\hline \multirow[t]{2}{*}{ Mean } & $37 \mathrm{~b}$ & $33 \mathrm{c}$ & $45 \mathrm{a}$ & $33 \mathrm{c}$ & 37 \\
\hline & \multicolumn{5}{|c|}{ Phosphorus levels $\left(\mathrm{g} \mathrm{kg}^{-1}\right)$} \\
\hline Turmalina & 2.5 & 2.3 & 4.1 & 3.5 & $3.1 \mathrm{~A}$ \\
\hline Cascata 968 & 2.8 & 2.0 & 3.5 & 3.4 & $2.9 \mathrm{~A}$ \\
\hline Cascata 848 & 2.4 & 2.3 & 3.8 & 3.4 & $3.0 \mathrm{~A}$ \\
\hline Precocinho & 2.7 & 2.7 & 4.6 & 3.5 & $3.4 \mathrm{~A}$ \\
\hline CP 9553 & 2.7 & 2.4 & 3.9 & 3.4 & $3.1 \mathrm{~A}$ \\
\hline СР 951 & 2.3 & 2.0 & 4.0 & 3.6 & $3.0 \mathrm{~A}$ \\
\hline Marli & 2.8 & 2.0 & 4.4 & 3.8 & $3.2 \mathrm{~A}$ \\
\hline Tropic Beauty & 2.2 & 2.1 & 4.0 & 14.2 & $5.6 \mathrm{~A}$ \\
\hline Sun Blaze & 2.6 & 2.3 & 4.3 & 3.8 & $3.2 \mathrm{~A}$ \\
\hline Jubileu & 2.7 & 2.2 & 3.8 & 3.3 & $3.0 \mathrm{~A}$ \\
\hline Mean & $2.6 \mathrm{~B}$ & $2.2 \mathrm{~B}$ & $4.0 \mathrm{AB}$ & $4.6 \mathrm{~A}$ & 3.4 \\
\hline
\end{tabular}

Means followed by different uppercase letters in the column and lowercase letters in the line differed according to Tukey's test at $5 \%$ significance. 
TABLE 2 - Potassium and calcium levels $\left(\mathrm{g} \mathrm{kg}^{-1}\right)$ in leaf samples of peach and nectarine cultivars at different harvests. Botucatu, 2009.

\begin{tabular}{|c|c|c|c|c|c|}
\hline \multirow[b]{2}{*}{ CULTIVARS } & \multicolumn{5}{|c|}{ HARVESTS } \\
\hline & 1 & 2 & 3 & 4 & Mean \\
\hline K & \multicolumn{5}{|c|}{ Potassium levels $\left(\mathrm{g} \mathrm{kg}^{-1}\right)$} \\
\hline Turmalina & $15 \mathrm{BCc}$ & $23 \mathrm{Ab}$ & $18 \mathrm{Ac}$ & $27 \mathrm{ABa}$ & $21 \mathrm{~A}$ \\
\hline Cascata 968 & $16 \mathrm{ABCc}$ & $19 \mathrm{ABbc}$ & $20 \mathrm{Aab}$ & $22 \mathrm{CDa}$ & $19 \mathrm{~A}$ \\
\hline Cascata 848 & $18 \mathrm{ABCab}$ & $17 \mathrm{ABb}$ & $17 \mathrm{ABab}$ & $21 \mathrm{Da}$ & $18 \mathrm{~A}$ \\
\hline Precocinho & $14 \mathrm{Cc}$ & $19 \mathrm{Bb}$ & $16 \mathrm{ABbc}$ & $23 \mathrm{BCDa}$ & $18 \mathrm{~A}$ \\
\hline СР 9553 & $19 \mathrm{ABb}$ & $18 \mathrm{ABb}$ & $20 \mathrm{Aab}$ & $23 \mathrm{ABCDa}$ & $20 \mathrm{~A}$ \\
\hline CP 951 & $18 \mathrm{ABCc}$ & $22 \mathrm{Ab}$ & $13 \mathrm{Bd}$ & $26 \mathrm{ABCa}$ & $20 \mathrm{~A}$ \\
\hline Marli & $15 \mathrm{ABCb}$ & $20 \mathrm{ABa}$ & $16 \mathrm{ABb}$ & $23 \mathrm{ABCDa}$ & $18 \mathrm{~A}$ \\
\hline Tropic Beauty & $20 \mathrm{Aab}$ & $16 \mathrm{Bb}$ & $18 \mathrm{Ab}$ & $22 \mathrm{CDa}$ & $19 \mathrm{~A}$ \\
\hline Sun Blaze & $18 \mathrm{ABCc}$ & $22 \mathrm{Ab}$ & $18 \mathrm{ABbc}$ & $27 \mathrm{Aa}$ & $21 \mathrm{~A}$ \\
\hline Jubileu & $17 \mathrm{ABCb}$ & $22 \mathrm{Aa}$ & $18 \mathrm{ABb}$ & $23 \mathrm{ABCDa}$ & $20 \mathrm{~A}$ \\
\hline \multirow[t]{2}{*}{ Mean } & $17 \mathrm{c}$ & $20 \mathrm{~b}$ & $18 \mathrm{c}$ & $24 \mathrm{a}$ & 20 \\
\hline & \multicolumn{5}{|c|}{ Calcium levels $\left(\mathrm{g} \mathrm{kg}^{-1}\right)$} \\
\hline Turmalina & $8 \mathrm{ABb}$ & $15 \mathrm{ABa}$ & $6 \mathrm{Ab}$ & $12 \mathrm{Aa}$ & $10 \mathrm{~A}$ \\
\hline Cascata 968 & $4 \mathrm{Bc}$ & $10 \mathrm{Ca}$ & $5 \mathrm{Abc}$ & $8 \mathrm{Aab}$ & $7 \mathrm{~B}$ \\
\hline Cascata 848 & $6 \mathrm{ABb}$ & $14 \mathrm{ABCa}$ & $6 \mathrm{Ab}$ & $11 \mathrm{Aa}$ & $9 \mathrm{AB}$ \\
\hline Precocinho & $5 \mathrm{ABc}$ & $15 \mathrm{ABCa}$ & $6 \mathrm{Ac}$ & $9 \mathrm{Ab}$ & $9 \mathrm{AB}$ \\
\hline CP 9553 & $5 \mathrm{ABb}$ & $13 \mathrm{ABCa}$ & $6 \mathrm{Ab}$ & $11 \mathrm{Aa}$ & $9 \mathrm{AB}$ \\
\hline СР 951 & $5 \mathrm{Bb}$ & $11 \mathrm{BCa}$ & $6 \mathrm{Ab}$ & $8 \mathrm{Aab}$ & $8 \mathrm{AB}$ \\
\hline Marli & $8 \mathrm{ABb}$ & $13 \mathrm{ABCa}$ & $8 \mathrm{Ab}$ & $8 \mathrm{Ab}$ & $9 \mathrm{AB}$ \\
\hline Tropic Beauty & $10 \mathrm{Ab}$ & $16 \mathrm{Aa}$ & $7 \mathrm{Ab}$ & $8 \mathrm{Ab}$ & $10 \mathrm{~A}$ \\
\hline Sun Blaze & $5 \mathrm{Bb}$ & $15 \mathrm{ABCa}$ & $6 \mathrm{Ab}$ & $12 \mathrm{Aa}$ & $9 \mathrm{AB}$ \\
\hline Jubileu & $6 \mathrm{ABc}$ & $13 \mathrm{ABCa}$ & $7 \mathrm{Abc}$ & $10 \mathrm{Aab}$ & $9 \mathrm{AB}$ \\
\hline Mean & $6 \mathrm{c}$ & $13 \mathrm{a}$ & $6 \mathrm{c}$ & $10 \mathrm{~b}$ & 9 \\
\hline
\end{tabular}

Means followed by different uppercase letters in the column and lowercase letters in the line differed according to Tukey's test at $5 \%$ significance. 
TABLE 3 - Magnesium and sulfur levels $\left(\mathrm{g} \mathrm{kg}^{-1}\right)$ in leaf samples of peach and nectarine cultivars at different harvests. Botucatu, 2009.

\begin{tabular}{|c|c|c|c|c|c|}
\hline \multirow{3}{*}{ CULTIVARS } & \multirow{2}{*}{\multicolumn{5}{|c|}{ HARVESTS }} \\
\hline & & & & & \\
\hline & 1 & 2 & 3 & 4 & Mean \\
\hline & \multicolumn{5}{|c|}{ Magnesium levels $\left(\mathrm{g} \mathrm{kg}^{-1}\right)$} \\
\hline Turmalina & $3.2 \mathrm{ABab}$ & $3.8 \mathrm{Ba}$ & $2.3 \mathrm{AB}$ & $3.7 \mathrm{Aa}$ & $3.2 \mathrm{AB}$ \\
\hline Cascata 968 & $2.3 \mathrm{Bab}$ & $3.2 \mathrm{Ba}$ & $2.1 \mathrm{ABb}$ & $3.0 \mathrm{Aab}$ & $2.7 \mathrm{~B}$ \\
\hline Cascata 848 & $2.9 \mathrm{ABab}$ & $3.5 \mathrm{Ba}$ & $2.4 \mathrm{ABb}$ & $3.7 \mathrm{Aa}$ & $3.1 \mathrm{AB}$ \\
\hline Precocinho & $2.6 \mathrm{Bbc}$ & $4.1 \mathrm{Ba}$ & $2.2 \mathrm{ABc}$ & $3.3 \mathrm{Aab}$ & $3.0 \mathrm{AB}$ \\
\hline CР 9553 & 2.7 Bab & $3.6 \mathrm{Bb}$ & $2.3 \mathrm{ABb}$ & $3.5 \mathrm{Ab}$ & $3.0 \mathrm{AB}$ \\
\hline СР 951 & $3.1 \mathrm{ABab}$ & 3.0 Bab & $2.3 \mathrm{ABb}$ & $3.3 \mathrm{Aa}$ & $2.9 \mathrm{AB}$ \\
\hline Marli & 4.0 Aa & 3.9 Bab & 3.2 Aab & $3.0 \mathrm{Ab}$ & $3.5 \mathrm{~A}$ \\
\hline Tropic Beauty & $3.3 \mathrm{ABb}$ & $5.5 \mathrm{Aa}$ & $2.4 \mathrm{ABb}$ & $2.8 \mathrm{Ab}$ & $3.5 \mathrm{~A}$ \\
\hline Sun Blaze & $2.5 \mathrm{Bb}$ & $3.9 \mathrm{Ba}$ & $2.0 \mathrm{Bb}$ & $3.6 \mathrm{Aa}$ & $3.0 \mathrm{AB}$ \\
\hline Jubileu & $2.8 \mathrm{Ba}$ & $3.2 \mathrm{Ba}$ & $2.5 \mathrm{ABa}$ & $2.8 \mathrm{Aa}$ & $2.8 \mathrm{AB}$ \\
\hline \multirow[t]{2}{*}{ Mean } & $2.9 \mathrm{~d}$ & $3.8 \mathrm{a}$ & $2.3 \mathrm{c}$ & $3.3 \mathrm{~b}$ & 3.1 \\
\hline & \multicolumn{5}{|c|}{ Sulfur levels $\left(\mathrm{g} \mathrm{kg}^{-1}\right)$} \\
\hline Turmalina & $1.7 \mathrm{Aa}$ & $1.3 \mathrm{ABb}$ & $1.7 \mathrm{BCa}$ & $1.3 \mathrm{Ab}$ & $1.5 \mathrm{ABC}$ \\
\hline Cascata 968 & $1.6 \mathrm{ABa}$ & $1.4 \mathrm{ABa}$ & $1.5 \mathrm{Ca}$ & $1.4 \mathrm{Aa}$ & $1.5 \mathrm{BC}$ \\
\hline Cascata 848 & $1.4 \mathrm{ABa}$ & $1.5 \mathrm{ABa}$ & $1.5 \mathrm{Ca}$ & $1.5 \mathrm{Aa}$ & $1.5 \mathrm{ABC}$ \\
\hline Precocinho & $1.5 \mathrm{ABb}$ & 1.7 Aab & $2.0 \mathrm{ABa}$ & $1.5 \mathrm{Ab}$ & $1.7 \mathrm{AB}$ \\
\hline CP 9553 & $1.4 \mathrm{ABb}$ & $1.5 \mathrm{ABab}$ & $1.8 \mathrm{BCa}$ & 1.6 Aab & $1.5 \mathrm{ABC}$ \\
\hline СР 951 & $1.2 \mathrm{Bb}$ & $1.4 \mathrm{ABab}$ & $1.6 \mathrm{BCa}$ & $1.3 \mathrm{Aab}$ & $1.4 \mathrm{C}$ \\
\hline Marli & $1.4 \mathrm{ABb}$ & $1.4 \mathrm{ABb}$ & $2.0 \mathrm{ABa}$ & $1.5 \mathrm{Ab}$ & $1.6 \mathrm{ABC}$ \\
\hline Tropic Beauty & $1.1 \mathrm{Bc}$ & $1.5 \mathrm{ABab}$ & $1.9 \mathrm{ABCa}$ & $1.5 \mathrm{Abc}$ & $1.5 \mathrm{ABC}$ \\
\hline Sun Blaze & $1.3 \mathrm{Bb}$ & $1.2 \mathrm{Bb}$ & $1.9 \mathrm{ABCa}$ & $1.4 \mathrm{Ab}$ & $1.4 \mathrm{BC}$ \\
\hline Jubileu & $1.3 \mathrm{ABb}$ & $1.6 \mathrm{ABb}$ & $2.3 \mathrm{Aa}$ & $1.6 \mathrm{Ab}$ & $1.7 \mathrm{~A}$ \\
\hline Mean & $1.4 \mathrm{~b}$ & $1.5 \mathrm{~b}$ & $1.8 \mathrm{a}$ & $1.5 \mathrm{~b}$ & 1.5 \\
\hline
\end{tabular}

Means followed by different uppercase letters in the column and lowercase letters in the line differed according to Tukey's test at $5 \%$ significance. 
TABLE 4 - Boron and copper levels $\left(\mathrm{mg} \mathrm{kg}^{-1}\right)$ in leaf samples of peach and nectarine cultivars at different harvests. Botucatu. 2009.

\begin{tabular}{cccccc}
\cline { 2 - 4 } & \multicolumn{5}{c}{ HARVESTS } \\
\hline CULTIVARS & 1 & 2 & 3 & 4 & Mean \\
\hline Turmalina & $67 \mathrm{ABb}$ & $50 \mathrm{Ab}$ & $130 \mathrm{Da}$ & $53 \mathrm{Ab}$ & $75 \mathrm{~B}$ \\
Cascata 968 & $93 \mathrm{Ab}$ & $46 \mathrm{Ac}$ & $135 \mathrm{CDa}$ & $51 \mathrm{Ac}$ & $81 \mathrm{AB}$ \\
Cascata 848 & $84 \mathrm{ABb}$ & $48 \mathrm{Ac}$ & $178 \mathrm{Ba}$ & $46 \mathrm{Ac}$ & $89 \mathrm{AB}$ \\
Precocinho & $75 \mathrm{ABb}$ & $51 \mathrm{Ab}$ & $133 \mathrm{Da}$ & $50 \mathrm{Ab}$ & $77 \mathrm{~B}$ \\
CP 9553 & $81 \mathrm{ABb}$ & $47 \mathrm{Ac}$ & $136 \mathrm{CDa}$ & $48 \mathrm{Ac}$ & $78 \mathrm{~B}$ \\
CP 951 & $76 \mathrm{ABb}$ & $56 \mathrm{Ab}$ & $171 \mathrm{BCa}$ & $50 \mathrm{Ab}$ & $88 \mathrm{AB}$ \\
Marli & $61 \mathrm{ABb}$ & $58 \mathrm{Ab}$ & $136 \mathrm{CDa}$ & $35 \mathrm{Ab}$ & $72 \mathrm{~B}$ \\
Tropic Beauty & $55 \mathrm{Bb}$ & $52 \mathrm{Ab}$ & $128 \mathrm{Da}$ & $62 \mathrm{Ab}$ & $74 \mathrm{~B}$ \\
Sun Blaze & $98 \mathrm{Ab}$ & $56 \mathrm{Ac}$ & $150 \mathrm{BCDa}$ & $54 \mathrm{Ac}$ & $89 \mathrm{AB}$ \\
Jubileu & $91 \mathrm{ABb}$ & $47 \mathrm{Ac}$ & $229 \mathrm{Aa}$ & $52 \mathrm{Ac}$ & $105 \mathrm{~A}$ \\
\hline Mean & $78 \mathrm{~b}$ & $51 \mathrm{c}$ & $152 \mathrm{a}$ & $50 \mathrm{c}$ & 83 \\
\hline Turmalina & $10 \mathrm{Ba}$ & $10 \mathrm{Aa}$ & $11 \mathrm{ABCa}$ & $10 \mathrm{ABCa}$ & $10 \mathrm{~A}$ \\
Cascata 968 & $11 \mathrm{Bab}$ & $10 \mathrm{Ab}$ & $14 \mathrm{Aa}$ & $9 \mathrm{ABCDb}$ & $11 \mathrm{~A}$ \\
Cascata 848 & $12 \mathrm{ABa}$ & $8 \mathrm{Ab}$ & $12 \mathrm{ABa}$ & $7 \mathrm{BCDb}$ & $10 \mathrm{~A}$ \\
Precocinho & $12 \mathrm{ABa}$ & $8 \mathrm{Ab}$ & $14 \mathrm{Aa}$ & $6 \mathrm{Db}$ & $10 \mathrm{~A}$ \\
CP 9553 & $15 \mathrm{Aa}$ & $8 \mathrm{Ab}$ & $14 \mathrm{Aa}$ & $6 \mathrm{Db}$ & $11 \mathrm{~A}$ \\
CP 951 & $11 \mathrm{ABa}$ & $9 \mathrm{Aa}$ & $9 \mathrm{BCa}$ & $11 \mathrm{ABa}$ & $10 \mathrm{~A}$ \\
Marli & $11 \mathrm{Ba}$ & $9 \mathrm{Aab}$ & $7 \mathrm{Cb}$ & $11 \mathrm{ABa}$ & $9 \mathrm{~A}$ \\
Tropic Beauty & $13 \mathrm{ABa}$ & $10 \mathrm{Aa}$ & $11 \mathrm{ABa}$ & $11 \mathrm{Aa}$ & $11 \mathrm{~A}$ \\
Sun Blaze & $13 \mathrm{ABa}$ & $8 \mathrm{Ab}$ & $14 \mathrm{Aa}$ & $8 \mathrm{ABCDb}$ & $11 \mathrm{~A}$ \\
Jubileu & $11 \mathrm{Bab}$ & $9 \mathrm{Abc}$ & $13 \mathrm{ABa}$ & $7 \mathrm{CDc}$ & $10 \mathrm{~A}$ \\
\hline Mean & $12 \mathrm{a}$ & $9 \mathrm{~b}$ & $12 \mathrm{a}$ & $9 \mathrm{~b}$ & 10 \\
\hline
\end{tabular}

Means followed by different uppercase letters in the column and lowercase letters in the line differed according to Tukey's test at $5 \%$ significance. 
TABLE 5 - Iron and manganese levels $\left(\mathrm{mg} \mathrm{kg}^{-1}\right)$ in leaf samples of peach and nectarine cultivars at different harvests. Botucatu. 2009.

\begin{tabular}{|c|c|c|c|c|c|}
\hline \multirow[b]{2}{*}{ CULTIVARS } & \multicolumn{5}{|c|}{ HARVESTS } \\
\hline & 1 & 2 & 3 & 4 & Mean \\
\hline & \multicolumn{5}{|c|}{ Iron levels $\left(\mathrm{mg} \mathrm{kg}^{-1}\right)$} \\
\hline Turmalina & $81 \mathrm{Ab}$ & $150 \mathrm{Aa}$ & $128 \mathrm{BCa}$ & $80 \mathrm{Ab}$ & $110 \mathrm{AB}$ \\
\hline Cascata 968 & $93 \mathrm{Aa}$ & $132 \mathrm{Aa}$ & $104 \mathrm{Ca}$ & $102 \mathrm{Aa}$ & $108 \mathrm{AB}$ \\
\hline Cascata 848 & $90 \mathrm{Ab}$ & $140 \mathrm{Aa}$ & $99 \mathrm{Cab}$ & $130 \mathrm{Aab}$ & $115 \mathrm{AB}$ \\
\hline Precocinho & $78 \mathrm{Ab}$ & $122 \mathrm{Aa}$ & $102 \mathrm{Cab}$ & $114 \mathrm{Aab}$ & $104 \mathrm{~B}$ \\
\hline СР 9553 & $76 \mathrm{Ac}$ & $122 \mathrm{Ab}$ & $168 \mathrm{Ba}$ & $124 \mathrm{Ab}$ & $123 \mathrm{AB}$ \\
\hline СР 951 & $70 \mathrm{Ac}$ & $118 \mathrm{Ab}$ & $170 \mathrm{Ba}$ & $88 \mathrm{Abc}$ & $112 \mathrm{AB}$ \\
\hline Marli & 79 Ac & $136 \mathrm{Ab}$ & $228 \mathrm{Aa}$ & $89 \mathrm{Ac}$ & $133 \mathrm{~A}$ \\
\hline Tropic Beauty & $77 \mathrm{Ac}$ & $122 \mathrm{Aab}$ & $125 \mathrm{BCa}$ & $82 \mathrm{Abc}$ & $101 \mathrm{~B}$ \\
\hline Sun Blaze & $70 \mathrm{Ab}$ & $124 \mathrm{Aa}$ & $93 \mathrm{Cab}$ & $104 \mathrm{Aab}$ & $98 \mathrm{~B}$ \\
\hline Jubileu & $102 \mathrm{Ab}$ & $152 \mathrm{Aa}$ & $105 \mathrm{Cb}$ & $116 \mathrm{Aab}$ & $119 \mathrm{AB}$ \\
\hline \multirow[t]{2}{*}{ Mean } & $82 \mathrm{c}$ & $132 \mathrm{a}$ & $132 \mathrm{a}$ & $103 \mathrm{~b}$ & 112 \\
\hline & \multicolumn{5}{|c|}{ Manganese levels $\left(\mathrm{mg} \mathrm{kg}^{-1}\right)$} \\
\hline Turmalina & 63 & 113 & 65 & 87 & $82 \mathrm{~B}$ \\
\hline Cascata 968 & 67 & 135 & 84 & 103 & $97 \mathrm{AB}$ \\
\hline Cascata 848 & 84 & 175 & 97 & 125 & $120 \mathrm{AB}$ \\
\hline Precocinho & 75 & 112 & 75 & 99 & $90 \mathrm{AB}$ \\
\hline CP 9553 & 53 & 142 & 67 & 85 & $87 \mathrm{AB}$ \\
\hline CP 951 & 75 & 80 & 94 & 87 & $84 \mathrm{AB}$ \\
\hline Marli & 65 & 129 & 73 & 78 & $86 \mathrm{AB}$ \\
\hline Tropic Beauty & 76 & 84 & 78 & 83 & $80 \mathrm{~B}$ \\
\hline Sun Blaze & 60 & 95 & 57 & 77 & $72 \mathrm{~B}$ \\
\hline Jubileu & 107 & 149 & 141 & 132 & $132 \mathrm{~A}$ \\
\hline Mean & $72 \mathrm{c}$ & $121 \mathrm{a}$ & $83 \mathrm{bc}$ & $96 \mathrm{~b}$ & 93 \\
\hline
\end{tabular}

Means followed by different uppercase letters in the column and lowercase letters in the line differed according to Tukey's test at $5 \%$ significance. 
TABELA 6 - Zinc levels $\left(\mathrm{mg} \mathrm{kg}^{-1}\right)$ in leaf samples of peach and nectarine cultivars at different harvests. Botucatu. 2009.

\begin{tabular}{cccccc}
\cline { 3 - 5 } & \multicolumn{5}{c}{ HARVESTS } \\
\hline CULTIVARS & 1 & 2 & 3 & 4 & Mean \\
\hline Turmalina & $14 \mathrm{ABb}$ & $15 \mathrm{Ab}$ & $27 \mathrm{Aa}$ & $26 \mathrm{Aa}$ & $21 \mathrm{~A}$ \\
Cascata 968 & $23 \mathrm{Aa}$ & $13 \mathrm{Ab}$ & $29 \mathrm{Aa}$ & $21 \mathrm{Aab}$ & $22 \mathrm{~A}$ \\
Cascata 848 & $15 \mathrm{ABbc}$ & $15 \mathrm{Ac}$ & $30 \mathrm{Aa}$ & $25 \mathrm{Aab}$ & $21 \mathrm{~A}$ \\
Precocinho & $16 \mathrm{ABc}$ & $12 \mathrm{Ac}$ & $38 \mathrm{Aa}$ & $27 \mathrm{Ab}$ & $23 \mathrm{~A}$ \\
CP 9553 & $25 \mathrm{Aa}$ & $15 \mathrm{Ab}$ & $32 \mathrm{Aa}$ & $25 \mathrm{Aa}$ & $24 \mathrm{~A}$ \\
CP 951 & $12 \mathrm{Bb}$ & $15 \mathrm{Ab}$ & $30 \mathrm{Aa}$ & $28 \mathrm{Aa}$ & $21 \mathrm{~A}$ \\
Marli & $16 \mathrm{ABc}$ & $12 \mathrm{Ac}$ & $38 \mathrm{Aa}$ & $25 \mathrm{Ab}$ & $23 \mathrm{~A}$ \\
Tropic Beauty & $18 \mathrm{ABc}$ & $14 \mathrm{Ac}$ & $38 \mathrm{Aa}$ & $27 \mathrm{Ab}$ & $24 \mathrm{~A}$ \\
Sun Blaze & $15 \mathrm{ABbc}$ & $12 \mathrm{Ac}$ & $29 \mathrm{Aa}$ & $22 \mathrm{Aab}$ & $19 \mathrm{~A}$ \\
Jubileu & $17 \mathrm{ABb}$ & $10 \mathrm{Ab}$ & $34 \mathrm{Aa}$ & $27 \mathrm{Aa}$ & $22 \mathrm{~A}$ \\
\hline Mean & $17 \mathrm{c}$ & $13 \mathrm{~d}$ & $32 \mathrm{a}$ & $25 \mathrm{~b}$ & 22 \\
\hline
\end{tabular}

Means followed by different uppercase letters in the column and lowercase letters in the line differed according to Tukey's test at $5 \%$ significance.

\section{CONCLUSIONS}

1. There were variations in the levels of $\mathrm{N}, \mathrm{Ca}, \mathrm{Mg}$, $\mathrm{S}, \mathrm{B}, \mathrm{Fe}$, and $\mathrm{Mn}$ for the different cultivars, with different responses according to the nutrient. As regards harvests at the different phenological stages of the peach tree, there was a difference in the leaf level of all evaluated nutrients.

2. In general, macronutrient uptake by the evaluated cultivars was in the following order: $\mathrm{N}>\mathrm{K}>\mathrm{Ca}>\mathrm{P}>\mathrm{Mg}>\mathrm{S}$. The order for micronutrient uptake was: $\mathrm{Fe}>\mathrm{Mn}>\mathrm{B}>\mathrm{Zn}>\mathrm{Cu}$.

\section{ACKNOWLEDGEMENTS}

This research project was supported by the FAPESP (Fundação de Amparo à Pesquisa do Estado de São Paulo). Process: 09/50862-3.

\section{REFERENCES}

ANUÁRIO BRASILEIRO DA FRUTICULTURA 2010. Santa Cruz do Sul: Editora Gazeta Santa Cruz do Sul, 2010. 128 p.

COMISSÃO DE FERTILIDADE DO SOLO - RS/ SC. Recomendação de adubação e calagem para os estados do Rio Grande do Sul e Santa Catarina. 3. ed. Passo Fundo: SBCS - Núcleo Regional Sul. 1994.
CROSSA-RAYNAUD, P., SOLEILLE, B., MARTINES-TELLES, J. Peach flowers sterility and its consequences on productivity. Acta Horticulturae, Leuven, n. 173, p. 93-102, 1985.

CUNHA, A. R.; KLOSOWSKI, E. S.; GALVANI, E. Classificação Climática para o município de Botucatu, SP, segundo Köppen. In: I SIMPÓSIO EM ENERGIA NAAGRICULTURA, 2., 1999, Botucatu. Anais...Botucatu: FCA, 1999. p. 490-491.

EMBRAPA. Centro Nacional de Pesquisa de Solos. Sistema brasileiro de classificação de solos. Rio de Janeiro, 1999. 412 p.

FACHINELLO, J.C., NATCHIGAL, J.C., KERSTEN, E. Fruticultura - Fundamentos e práticas. Pelotas: Editora Universitária-UFPEL, 311p. 1996. Freire, C.J.S., Magnani, M. Adubação e correção do solo. In: Raseira, M.C.B., Centellas-Quezada, A. (Eds). Pêssego - Produção. Brasília: Embrapa Informação Tecnológica, 2003. p. 77-89.

GAO, D.S., LI, X.L., SHU, H.R. Effects of chemical defoliation on the endodormancy of peach trees. Journal of Fruit Science. Baton Rouge, v. 19, n. 4, p. 269-271, 2002. 
JOHNSON, R.S., URIU, K. Mineral Nutrition. In: Larue, J.H., Johnson, R.S. (eds.). Peaches, Plums and Nectarines growing and handling for fresh market. University of California, Division of Agriculture and Natural resources, Pub. 3331. P. 68-81, 1989.

JOHSON, R.S., ANDRIS, H., DAY, K., BEEDE, R. Using dormant shoots to determine the nutritional status of peach trees. Acta Horticulturae, Leuven, v. 721, p. 285-290. 2006.

LEECE, D.R., BARKUS, B. Diagnostic leaf analysis for stone fruits. Nutritional status of peach orchards. Australian Journal of Experimental Agriculture and Animal Husbandry, Armidale, v. 14, n. 71, p. 828-834, 1974.

MALAVOLTA, E. Elementos de nutrição mineral de plantas. São Paulo: Ceres, 1980. 251 p.

MALAVOLTA, E., VITTI, G.C., OLIVEIRA, S.A. Avaliação do estado nutricional das plantas: princípios e aplicações. POTAFOS: Piracicaba, 2 ed. 1997.319 p.

MATTOS, M.L.T., FREIRE, C.J.S., MORAES, E.C. Crescimento e teores foliares de N, P, K, Ca e Mg em pessegueiro cv. Diamante com diferentes níveis de $\mathrm{N}$ aplicado ao solo. Pesquisa Agropecuária Brasileira, Brasília, v. 26, n. 9, p. 1315-1321, 1991.

NAVA, G.A., MARODIN, G.A.B., SANTOS, R.P. Reprodução do pessegueiro: efeito genético, ambiental e de manejo das plantas. Revista Brasileira de Fruticultura, Jaboticabal, v. 31, n. 4, p. 12181233, 2009.
RAIJ, B. VAN,; QUAGGIO, J. A. Métodos de análise de solo para fins de fertilidade. Campinas: Instituto Agronômico de Campinas, 1983. (Boletim Técnico, 81).

ROMBOLÁ, A.D., TOSELI, M., SCUDELLAR, D. A nutrição de frutas de caroço na fruticultura ecocompatível, IN: SIMPÓSIO INTERNACIONAL DE FRUTAS DE CAROÇO PÊSSEGOS, NECTARINAS E AMEIXAS, 2000, Porto Alegre. Anais... Porto Alegre, RS, UFRS, p. 41-60, 2000.

PEREIRA, F.M., NATCHIGAL, J.C., ROBERTO, S.R. Tecnologia para a cultura do pessegueiro em regiões tropicais e subtropicais. Jaboticabal: FUNEP, 2002. 62 p.

SERRAT, B.M., REISMANN, C.B., MOTTA, A.C.V., MARQUES, R. Nutrição mineral de fruiteras de caroço. In: Monteiro, L.B., May-De Mio, L.L., Serrat, B.M., Cuqyel, F.L. Fruteiras de caroço: uma visão ecológica, Curitiba: UFPR, 2004, p. 71-95.

WOLUKAU, J.N., ZHANG, S., XU, G., CHEN, D. The effect of temperature, polyamines and polyamine synthetics inhibitor on in vitro pollen germination and pollen tube growth of Prunnus mume. Scientia Horticulturae, Amsterdan, v. 99, n. 3-4, p. 289-299, 2004. 